\title{
ESISTE: Supporting Inclusion of Students with Special Needs in Mainstream Classrooms
}

\author{
Barbara Rita Barricelli1 ${ }^{10000-0001-9575-5542]}$, Alessandro Rizzi ${ }^{1[0000-0001-6240-4383]}$, \\ Stefano Valtolina ${ }^{10000-0003-1949-2992]}$, Claudio Manfredini ${ }^{2}$ \\ ${ }^{1}$ Dept. of Computer Science, Università degli Studi di Milano, 20135 Milano, Italy \\ ${ }^{2}$ I.I.S. Piero Sraffa, 26013 Crema, Italy \\ barricelli@di.unimi.it
}

\begin{abstract}
This chapter presents the outcomes of the first stage of ESISTE ESISTE (Expert System Interaction Structured To Education), an interdisciplinary research project aimed at designing and developing an interactive system for the management of ICF-CY classification (International Classification of Functioning, Disability and Health - Children and Youth) in primary, secondary, and higher education levels in Italy. The goal of the project is to enable the inclusion in the education system of students with special needs involving in the process their teachers, family members, close friends, healthcare professionals and other stakeholders.
\end{abstract}

Keywords: ICF Classification, ICF-CY, Functioning, Disability, Special Needs, Health, Education, Collaboration, Teaching, Human Work Interaction Design, Mainstream classrooms.

\section{Introduction}

The International Classification of Functioning, Disability and Health (ICF) is a classification defined by the World Health Organization (WHO) in 2001 for providing a standard language and framework to describe health and health-related components of well-being (e.g., education, labour) [1]. In 2007, WHO published a further version of ICF specific for children and youth, called ICF-CY, from birth to 17 years of age. The ICF classification uses three main codes to describe an individual: Body functions and structures, activities and participation, and environmental factors. The ICF-CY classification is in use in Italian education system since 2007 but there is not yet a complete and widely-used interactive system to support its use and what is more important, there are no tools to support the collaboration between all the stakeholders involved in the student's evaluation. Healthcare professionals and teachers most of the times use different systems for ICF management (mostly paper based) and in order to exchange their views on a student they need to physically meet and/or send digitalized documents and discuss them over the phone or via email. One of the reasons why ICF-CY classification is adopted in educational systems is that it supports the stakeholders to identify special needs in students. 
In literature, a number of studies discussing the inclusion of special needs students in mainstream classrooms (e.g., [2][3][4][5]). The main idea is that inclusion may help the students in integrating themselves in society and in dealing with their difficulties and eventual disadvantages.

This chapter presents the outcomes of the first stage of ESISTE (Expert System Interaction Structured To Education), a National project that aims at exploiting ICF-CY potentials for facilitating the inclusion of students with special needs in mainstream (i.e. non dedicated)classrooms. To this aim, the authors designed and developed an interactive high-fidelity prototype for using the ICY-CY classification by empowering all stakeholders; according to the authors' analysis, teachers, clinicians, and system administrators are the three user groups that have to be considered. Given the deep diversity of these three classes of users, the Human Work Interaction Design (HWID) discipline [6][7][8][9] helped in analyzing the user and functional requirements addressing their needs.

The chapter is organized as follows. Section 2 presents the ICF-CY classification. Section 3 illustrates the ESISTE Project. In Section 4, the design and development activities for the creation of an interactive prototype are presented, while in Section 5 the results of usability evaluation are illustrated and discussed.

\section{ICF-CY}

The International Classification of Functioning, Disability and Health [1] defines a classification for describing the state of health of people in relation to their community environments (social, family, work context) for grasping the difficulties due to the socio-cultural context of reference that can cause disability. The International Classification of Functioning, Disability and Health - Version for Children and Adolescents (ICFCY[10] provides precise descriptions of the health status of children and youth. As a derived classification, it includes further detailed information on the application of the ICF when documenting the relevant aspects of functioning and health in children and youth.

The ICF-CY shares the same classification properties with its reference classification the ICF and offers a common and standardized language to describe and measure health and disability up to eighteen years of life. This classification system helps clinicians, teachers, researchers, administrators, policy makers and parents to identify the health, development and education children and youth's needs.

In practice, the ICY-CY is a model that describes different children and youth's levels of ability in functional areas, such as learning, mobility, communication, self-care, social relationships, and other similar characteristics. It encourages the development of interventions that targets at the development of individual' functioning in relation to their environment and personal conditions. 
According to the model depicted in Figure 1, the ICF-CY functioning codes can be classified in (i) body functions (i.e., physiological and psychological), (ii) body structures (i.e., anatomical parts such as organs, limbs and their components), (iii) activities (i.e., execution of a task or action), and (iv) participation that is the involvement in life situations such as learning, self-care, communication, relationships or participation in school activities [10]. Contextual factors interact with the individual with a health condition and determine the level and extent of the individual's functioning. Environmental factors are extrinsic to the individual and can related to the social support, relations, the attitudes of the society, the legal system. Personal Factors (e.g. gender, race, age, lifestyle, habits) do not have categories within the ICF-CY, and open to the subjective view of the users [10].

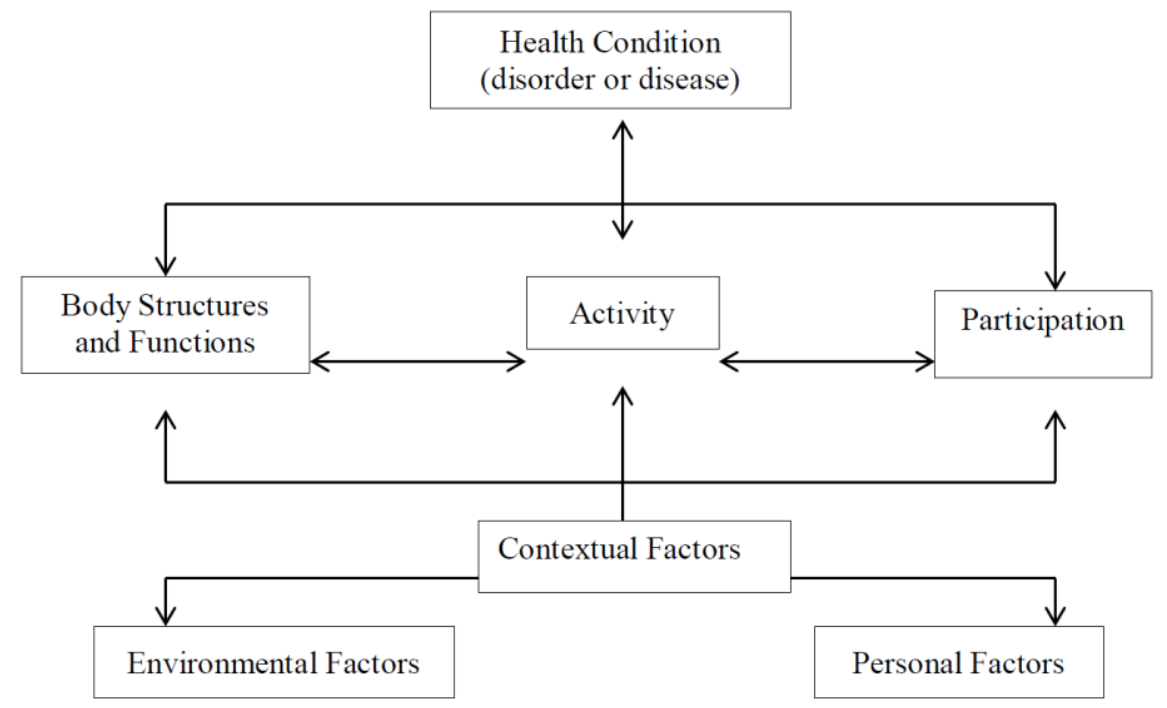

Fig. 1. Interactions between the components of the ICF-CY Model [11].

\section{The ESISTE Project}

The ESISTE (Expert System Interaction Structured To Education) Project has as main goal to identify the difficulties related to ICF model complexity of use. The need is to simplify the use of ICF model as a tool for supporting special needs students' inclusion. The idea is to create an interactive system to be used in Italian schools in all of its levels of instruction: primary (elementary schools), secondary (middle school) and higher education (high school). The system would be aimed at understanding which inclusion approaches to apply with students with special needs on the basis of body functions and structure, activities and participation, environmental and personal factors, and other information related to the severity of their disability and their age.

The seven main objectives of the ESISTE Project are: 
1. to analyze, in the various context diversified by instruction level, which types of approach are more favorable for the students' integration in relation to bodily functions, activities and participation, environmental factors and the type of disability;

2. to study how to retrieve information related to contextual factors that act as facilitators in the process of inclusion of the students in classrooms;

3. to adapt the ICF classification in the perspective of a guided and finalized use to the proposition of didactic paths, educational tools or integrative activities;

4. to realize a dynamic and functional tool, which can act as facilitator and catalyst of the individual's attention and at the same time as data detector by the teacher (i.e., the definition of the expert system) using the classification scheme ICF-CY;

5. to use the expert system for detecting needs and proposing inclusive strategies with a view to improving the performance of the student in the school environment and in the social sphere;

6. to propose an innovative and accessible man-machine interaction modality as a facilitator in the breaking down of barriers in situations of limitation of activities and participation;

7. to implement a network approach, which can involve not only the school environment but also all the other stakeholders that during the life of a disabled person come into play.

The ESISTE system will be used by a network of stakeholders - a Community of Interest [12] - constituted by all the people who are involved in the life of a student with special needs, with different roles, in different times and in different ways, not only related with the school environment. The use of a shared tool would help in designing an ad-hoc education plan focused on inclusion and education and will enable family members, healthcare assistants, close friends and teachers to collaborate. Collecting evaluations based on the ICF classification and allowing the stakeholders to compare the different evaluations on the same students will help them to consider all the different points of view on the issues that can affect the student's life at school and her/his learning process.

The final goal of the ESISTE Project aims at designing a shared tool that provides a screening for pupil inclusion according to ICF-CY parameters and able to support integrative teaching actions for the formulation of functional diagnosis and individualized educational plans. The idea is to simplify the use of the ICF-CY dropping it into the school realities of different orders and degrees through the use of an usable expert software system accessible according to the Italian Stanca $\mathrm{Act}^{1}$ (AA according to the W3C standard).Through a set of auxiliaries and integrative strategies, this shared virtual environment allows teachers, parents and clinician specialists to follow pupils with special needs during their scholar paths, from primary school through graduate school. The result is the definition of guidelines to use for the implementation a real life plan that takes into account all ICF aspects from the body condition to the contextual factors. Teachers, parents and other collaborators can actively cooperate in the design of these

${ }^{1}$ The Italian accessibility law: Stanca Act http://www.agid.gov.it/dm-8-luglio-2005-allegato-A 
paths with the aim to achieve better performance in different areas of their sons and pupil's life.

This chapter presents the outcomes of a first stage of the ESISTE Project, aimed at participatory designing a prototype of the user interface for the interactive system. Particularly, the authors capitalized on their experience in Cultures of Participation, Participatory Design and Collaboration at large (e.g., [13][14][15][16][17]) and focused their work on the Human Work Interaction Design aspects of the Project: this chapter presents in fact the participatory design approach and the usability evaluation of a prototype.

\section{Design and Development of ESISTE Prototype}

In the definition of the requirements' specification the authors considered two main aspects: the profiles of the users who will use the system and the necessary functional requirements, which strictly depend on the type of user. For this first stage of the ESISTE project, three main types of users have been identified and have been involved in a participatory design process and in further usability evaluations:

- Teacher: Differentiated by education level (primary, secondary and higher education), they know the student, her/his special needs and education history so far.

- Clinician: Typically, a neuropsychiatrist, knows the student and starts to fill the ICF checklist creating a subset of parameters that are suitable for her/his profile. The clinician is usually the one who plays the facilitator role in managing the relationships with all the stakeholders involved.

- System administrator: Typically, a technician who works regularly with inclusive technology and contributes to the removal of barriers.

Several meetings with one or more representatives of these three user groups have been organized and they have been invited in discussing about expected metaphor and style of interaction and also in the preliminary design of a paper prototype. The outcomes of these meetings allowed to proceed in the preparation of a first interactive vertical prototype that has been tested with a predictive method. The results of this first evaluation led to the development of an improved prototype that has been tested involving representatives of the actual final users. A screenshot of the prototype is depicted in Fig. 2. The system allows to assign several evaluators to each student and all evaluators are able to compare their ratings with the ones of the other evaluators (see Fig. 3). 


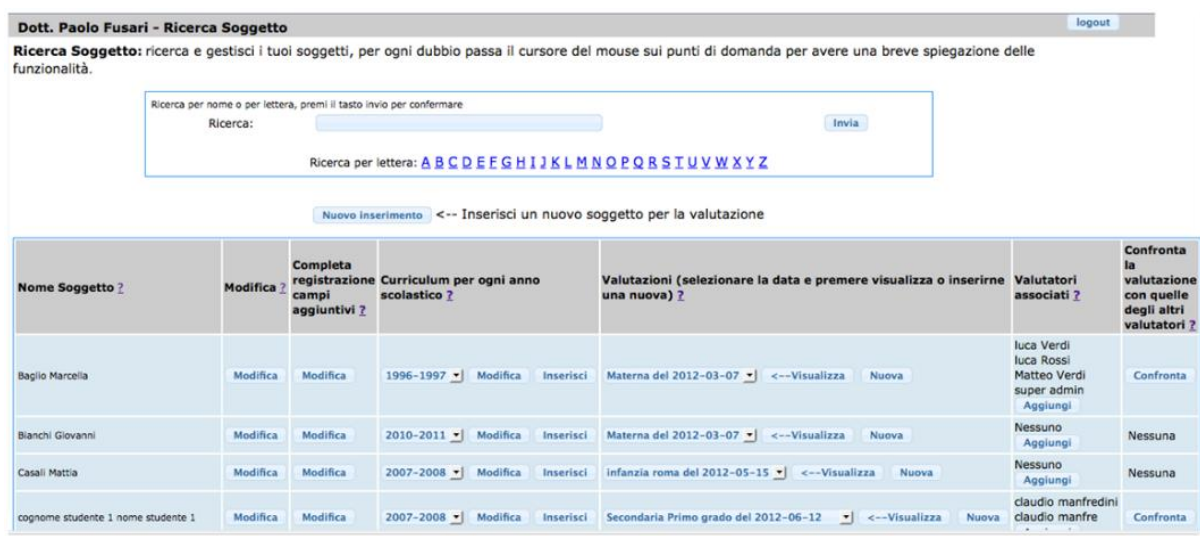

Fig. 2. A clinician accesses the system for rating some students.

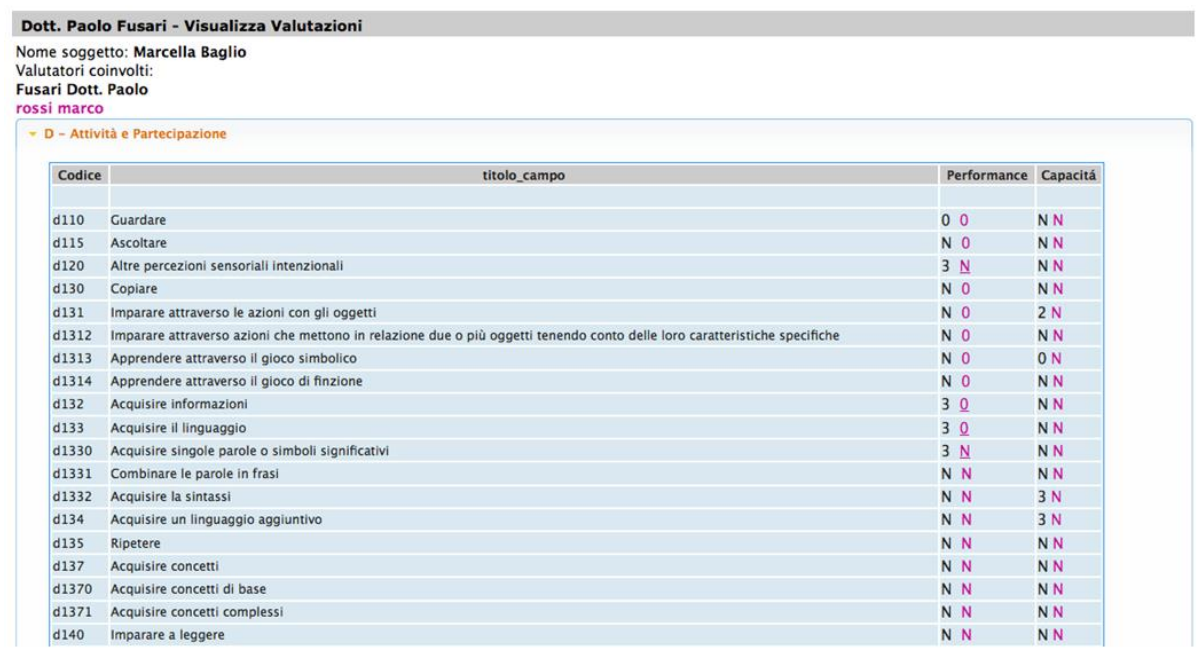

Fig. 3. A teacher accesses a students' evaluation and compares his ratings with the ones of the clinician.

\section{$5 \quad$ Usability Evaluation}

The heuristic evaluation method [18] has been applied to test the usability of the first prototype. Four usability experts have been involved in the evaluations. For the evaluations the authors applied the ten Nielsen's heuristics: 1) Visibility of system status; 2) Match between system and real world; 3) User control and freedom; 4) Consistency and standards; 5) Error prevention; 6) Recognition rather than recall; 7) Flexibility and efficiency of use; 8) Aesthetic and minimalist design; 9) Help users recognize, diagnose, and recover from errors; 10) Help and documentation. The Nielsen's severity ranking [19] has been used to classify the usability issues: 1) Cosmetic problem only; 
2) Minor usability problem; 3) Major usability problem; and 4) Usability catastrophe. The results of the heuristic evaluation highlighted the need of a deep improvement of the prototype. Fig. 4 shows the number of violations for each heuristic.

The improvements have been illustrated and further discussed in a meeting that involved both the developers and the final users' representatives and informed the preparation of the second interactive prototype that has been tested with the final users. The authors invited to the participatory design activities 7 people, all ICF expert users, who usually collaborate in Italian schools taking care of several students with special needs: 5 teachers (working at different education level), 1 clinician, and 1 system administrator.

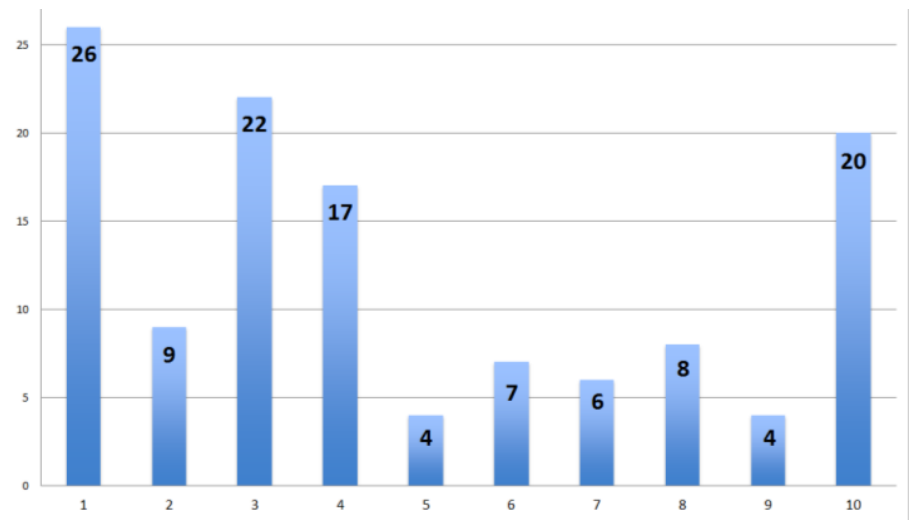

Fig. 4. For each of the 10 Nielsen's heuristics the chart shows the number of violations detected during the usability analysis.

The participants have been provided with an initial demographic questionnaire aimed at knowing better their profile and skills in using interactive systems for work or personal matters. The results are presented in Figure 5.

Different tasks have been assigned to the participants on the basis of their role. The system administrator has been asked to register the other participants as evaluators and a student in the ESISTE system. The clinician has been invited to complete the student evaluation by using the ICF classification tool. The teachers have been asked to complete their own evaluation of the student and to compare their evaluation with the ones registered by other colleagues. To conclude the user test the participants were asked to fill in two final questionnaires, SUS (System Usability Scale) [20] and CSUQ (Computer System Usability Questionnaire) [21], and to respond to two open-ended questions. SUS is a usability questionnaire very broadly used, especially in industry that can return reliable results even when administered to small sample of users. CSUQ is a questionnaire developed by IBM and is mostly focused on measuring the satisfaction in using the application or tool under evaluation. 

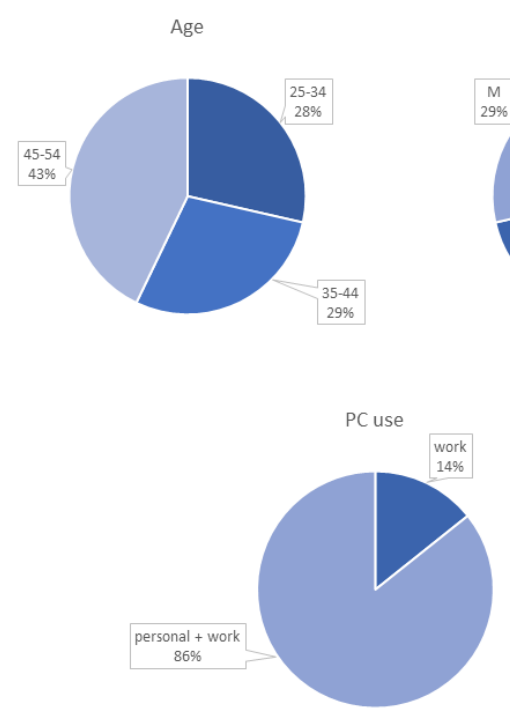

Are you familiar with ICF-CY?

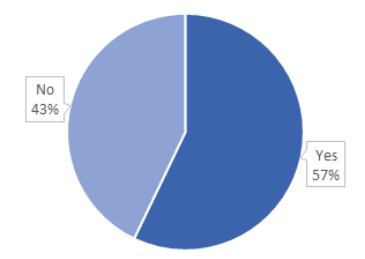

Gender

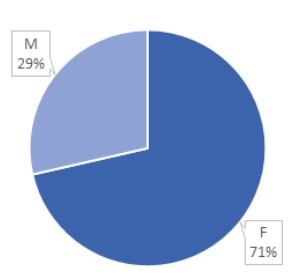

Job

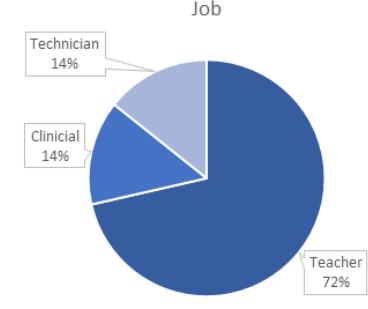

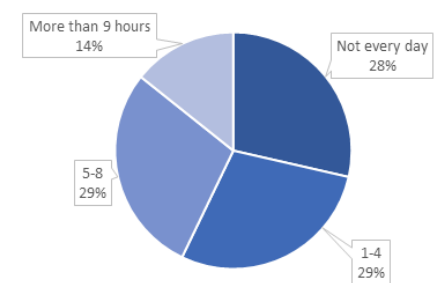

How often do you use ICF-CY?

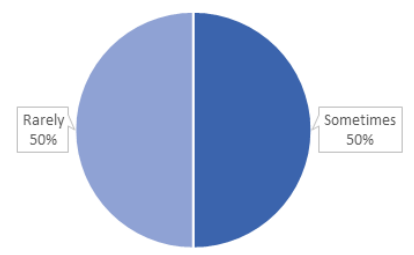

Fig. 5. The results of the initial demographic questionnaire.

The SUS questionnaire results are presented in Table 1. The average is 67.14 and is slightly below the average as defined by the creators of the SUS method (that is 68).

To interpret the CSUQ questionnaire results, these four factors were used: System Usefulness (SYSUSE), Information Quality (INFOQUAL), Interface Quality (INTERQUAL), and Overall Satisfaction (OVERALL). The minimum value for the factors is 1 and the maximum is 5 . The results, presented in Table 2, show that the system has been evaluated above the average for all the four factors considered. 
Table 1. The results of the SUS final questionnaire. .

\begin{tabular}{ll}
\hline Participant & SUS \\
\hline System administrator & 70 \\
Clinician & 60 \\
Teacher 1 & 72.5 \\
Teacher 2 & 77.5 \\
Teacher 3 & 67.5 \\
Teacher 4 & 62.5 \\
Teacher 5 & 60 \\
Average & 67.14 \\
\hline
\end{tabular}

The first of the two open-ended questions was about the comparison of this system with other existing systems for ICF classification. None of the participants is familiar with other systems. The second question asked the participants to describe what and how the system should be changed to better meet their needs and expectations. Six out of seven participants responded to this question and asked the following enhancements: a help tool for the ones who are still not familiar with the ICF classification, and an online chat tool and sticky notes to discuss the evaluations in real time and asynchronously with the colleagues.

Table 2. The results of the CSUQ final questionnaire.

\begin{tabular}{lllll}
\hline Participant & SYSUSE & INFOQUAL & INTERQUAL & OVERALL \\
\hline System administrator & 3 & 2.8 & 3.5 & 4 \\
Clinician & 3 & 3.3 & 3.5 & 3.1 \\
Teacher 1 & 3 & 3.7 & 4 & 4 \\
Teacher 2 & 4.5 & 3.8 & 4 & 4.1 \\
Teacher 3 & 3.5 & 3.7 & 3.5 & 3.9 \\
Teacher 4 & 4 & 3.3 & 4 & 3.1 \\
Teacher 5 & 3 & 3.2 & 3 & 3.2 \\
Average & 3.4 & 3.4 & 3.6 & 3.6 \\
\hline
\end{tabular}




\section{Conclusions and Future Developments}

This first stage of the ESISTE project was focused mainly on the participatory design of a prototype of the system. Final users of the system (clinicians, technicians and teachers) were involved to a participatory design process that informed the authors about the ICF-CY classification and its adoption in primary, secondary and higher education in Italy. After this first stage of the project, the prototype will be further developed leading to the creation of a full system to be tested on large scale in different schools and with real use cases. New users' representatives will be involved, like family members and close friends whose contribution will be fundamental in the correct implementation of the ESISTE system and process of collaboration.

\section{References}

1. World Health Organization: International Classification of Functioning, Disability and Health. World Health Organization, Geneve (2001).

2. Wang, H.L.: Should All Students with Special Educational Needs (SEN) Be Included in Mainstream Education Provision? - A Critical Analysis. International Education Studies 2(4), 154-161 (2009).

3. Hanline, M.F.: Inclusion of Preschoolers with Profound Disabilities: An Anlaysis of Children's Interactions. Journal of the Association for Persons with Severe Handicaps, 18(1), 28-35 (1993).

4. Peterson, N.L., Haralick, J.G.: Integration of Handicapped and Non-Handicapped Preschoolers: An Analysis of Play Behavior and Social Interaction. Education and Training of the Mentally Retarded 12, 235-245 (1977).

5. Jenkinson, J.C.: Mainstream or Special? Educating Students with Disabilities. London: Routledge (1997).

6. Abdelnour-Nocera, J, Barricelli, B.R., Lopes, A., Campos, P., Clemmensen, T.: Preface. In: IFIP Advances in Information and Communication Technology, 468. Springer New York LLC (2015). ISBN: 9783319270470.

7. Campos, P., Clemmensen, T., Abdelnour-Nocera, J., Katre, D., Lopes, A., Ørngreen, R. (Eds.): Human Work Interaction Design. Work Analysis and HCI. Springer-Verlag Berlin Heidelberg (2013).

8. Clemmensen, T., Campos, P., Ørngreen, R., Pejtersen, A. M., Wong W. (Eds.): HumanWork Interaction Design: Designing for Human Work. Springer US (2006).

9. Katre, D., Ørngreen, R., Yammiyavar, P., Clemmensen, T. (Eds.): Human Work Interaction Design: Usability in Social, Cultural and Organizational Contexts. Springer-Verlag Berlin Heidelberg (2010).

10. World Health Organization. International Classification of Functioning, Disability and Health: Children \& Youth Version: ICF-CY. Retrieved from: apps.who.int/iris/bitstream/10665/43737/1/9789241547321_eng.pdf

11. Faracul, F.E.: Implementing the Biopsychosocial Approach of the ICF-CY to Predict Secondary Conditions and Quality of Life in Individuals with Spina Bifida. Dissertation at The University of North Carolina at Chapel Hill (2017).

12. Fischer, G.: Communities of interest: Learning through the interaction of multiple knowledge systems. In: Proceedings 24th IRIS Conference. Department of Information Science. Bergen (2001). 
13. Barricelli, B. R., Fischer, G., Mørch, A., Piccinno, A., Valtolina, S.: Cultures of participation in the digital age: Coping with information, participation, and collaboration overload. LNCS, 9083, pp. 271-275. Springer Verlag. (2015). DOI: 10.1007/978-3-319-18425-8_28.

14. Barricelli, B. R., Gheitasy, A., Mørch, A., Piccinno, A., Valtolina, S.: Culture of participation in the digital age: Social computing for learning, working, and living. In: Proceedings of the Workshop on Advanced Visual Interfaces AVI, pp. 387-390. ACM. (2014). DOI: 10.1145/2598153.2602223.

15. Barricelli, B.R., Fischer, G., Fogli, D., Mørch, A., Piccinno, A., Valtolina, S.: Cultures of Participation in the Digital Age: From "Have to" to "Want to" Participate. In: Proc. NordiCHI '16, 1-3 (2016).

16. Valtolina, S., Barricelli, B.R., Dittrich, Y.: Participatory Knowledge-Management Design: a Semiotic Approach. Journal of Visual Languages and Computing (JVLC), 23(2), 103-115 (2011).

17. Zhu, L., Mussio, P., Barricelli, B.R., Iacob, C.: A Habitable Space for Supporting Creative Collaboration. In: Proc. CTS2010, 617-622, IEEE Computer Society (2010).

18. Nielsen, J., Molich, R.: Heuristic evaluation of user interfaces. In: Proc. CHI 1990, pp.249256. ACM Press, New York (2010).

19. Nielsen, J., Mack R.: Usability inspection methods. Wiley, New York (1994).

20. Brooke, J.: SUS: a "quick and dirty" usability scale. In: Usability Evaluation in Industry, 189-194. Taylor and Francis (1996).

21. Lewis, J. R.: IBM Computer Usability Satisfaction Questionnaires: Psychometric Evaluation and Instructions for Use. International Journal of Human-Computer Interaction 7(1), 57-78 (1995). 